\title{
Growth of a two-phase finger in eutectics systems
}

\author{
G. Boussinot, ${ }^{1}$ C. Hüter, ${ }^{2}$ and E. A. Brener ${ }^{1}$ \\ ${ }^{1}$ Institut für Festkörperforschung, Forschungszentrum Jülich, D-52425 Jülich, Germany \\ ${ }^{2}$ Max-Planck-Institut für Eisenforschung, D-40237 Düsseldorf, Germany
}

(Received 16 November 2010; published 8 February 2011)

\begin{abstract}
We present a theoretical study of the growth of a two-phase finger in eutectic systems. This pattern was observed experimentally by Akamatsu and Faivre [Phys. Rev. E 61, 3757 (2000)]. We study this two-phase finger using a boundary-integral formulation and we complement our investigation by a phase-field validation of the stability of the pattern. The deviations from the eutectic temperature and from the eutectic concentration provide two independent control parameters, leading to very different patterns depending on their relative importance. We propose scaling laws for the velocity and the different length scales of the pattern.
\end{abstract}

DOI: 10.1103/PhysRevE.83.020601

PACS number(s): 68.70.+w, 64.70.kd

One of the most common modes of growth of a solid phase from a metastable liquid is the dendritic one where a nearly parabolic front advances at a constant velocity. The possibility of a steady-state growth of a parabolic front was first demonstrated by Ivantsov [1] and was supplemented by the determination of the anisotropy of surface tension as a selection mechanism for the velocity (see, for example, [2,3] and references therein). Recently, selection mechanisms such as the presence of a triple junction [4,5] or of elastic effects [6] were reported.

Another classical mode of solidification is the lamellar growth in eutectic alloys. The pioneering work of Jackson and Hunt [7] on this topic refers to directional solidification and especially consists of finding the temperature of the solidification front. In opposition to dendritic growth, no unique solution exists for this process. A range of lamellae spacing is stable and bifurcations toward a broad range of oscillatory regimes [8-10] or tilted patterns $[11,12]$ have been evidenced. It is worthwhile to mention the recent observation of a three-dimensional spiral dendrite in eutectics [13].

Despite a large amount of theoretical and experimental studies on eutectics, the problem of dendritic patterns has, to our knowledge, never been addressed on a theoretical level in these systems. However a dendritelike structure called "two-phase fingers" (see Fig. 7 in [14]) has been observed by Akamatsu and Faivre for an off-eutectic concentration and it is suggested that such a pattern could grow with a constant velocity in directional experiments. This is what we study here in the isothermal case, that is, a two-dimensional (2D) solidifying dendrite with one solid phase surrounded by the other one. The framework of the boundary-integral technique is used and we supplement our results with a phase-field calculation showing that the eutectic two-phase finger is a stable mode of growth. We discuss our results giving plausible arguments for the scaling of physical quantities.

Geometry and phase diagram. The 2D dendritelike eutectic finger studied in this article consists of two different solid phases growing at the expense of the metastable liquid. The exterior part consists of a first solid, called ext, which exhibits an Ivantsov parabolic front asymptotically far from the tip. The interior part consists of a lamella of a second solid, called int, the lamella being parallel to the direction of velocity (see Fig. 1). We denote by $2 a$ the width of this interior lamella. In Fig. 2, we present the corresponding phase diagram. The operating point (temperature $T_{0}$ and concentration $C_{\infty}$ ), indicated by the black circle, lies in the liquid-ext two-phase region. $C_{\text {Sext }}\left(C_{\text {Sint }}\right)$ is the concentration of the solid ext (int) in equilibrium with the liquid $L$ and $C_{\text {Lext }}\left(C_{\text {Lint }}\right)$ is the concentration of the liquid $L$ in equilibrium with solid ext (int).

Boundary-integral formulation. The data about the phase diagram and the operating point enter the boundary-integral formulation of the problem. It consists of writing down one integro-differential equation for each solid-liquid interface having additional constraints at the triple junction. The equations for the solid-liquid interfaces satisfy the steadystate bulk diffusion equation and incorporate appropriate boundary conditions. The boundary-integral technique for the study of eutectic systems has been widely developed and used, and we refer to [15] for a detailed derivation of the equations. Here, instead of the periodic boundary conditions used in lamellar growth, our two-phase finger has to correspond to an Ivantsov parabola far behind the tip. The radius of curvature $\rho$ of this parabola enters the definition of the Peclet number $P=V \rho / 2 D$, where $V$ is the steady-state velocity of the finger and $D$ is the impurity diffusion coefficient. The Peclet number is related to the composition of the metastable liquid $C_{\infty}$ through the Ivantsov relation. We measure the lengths $x$ and $y$ of our system of coordinates in units of $a$, the width of the interior lamella, and define $\lambda=a / \rho$. Then the equation for the solid-liquid interface $y_{i}(x)(i=$ int or $i=$ ext $)$ reads

$$
\begin{aligned}
& -\frac{1}{2} \Delta_{L} \delta_{i, \text { int }}-\frac{1}{2}\left[1-(t+1) \delta_{i, \mathrm{int}}\right] \frac{d_{0}}{a} \kappa\left[y_{i}^{\prime}\left(x^{\prime}\right)\right] \\
& =-\Delta_{\infty}-\frac{P \lambda}{\pi} t \int_{0}^{1} d x g\left[x, y_{\mathrm{int}}(x) ; x^{\prime}, y_{i}^{\prime}\left(x^{\prime}\right)\right] \\
& \quad+\frac{P \lambda}{\pi} \int_{1}^{\infty} d x g\left[x, y_{\mathrm{ext}}(x) ; x^{\prime}, y_{i}^{\prime}\left(x^{\prime}\right)\right]+\frac{P \lambda}{2 \pi} \\
& \quad \times \int_{0}^{1} d x\left\{\Delta_{L}-t \frac{d_{0}}{a} \kappa\left[y_{\mathrm{int}}(x)\right]\right\} g^{\prime}\left[x, y_{\mathrm{int}}(x) ; x^{\prime}, y_{i}^{\prime}\left(x^{\prime}\right)\right] \\
& \quad+\frac{P \lambda}{2 \pi} \int_{1}^{\infty} d x \frac{d_{0}}{a} \kappa\left[y_{\mathrm{ext}}(x)\right] g^{\prime}\left[x, y_{\mathrm{ext}}(x) ; x^{\prime}, y_{i}^{\prime}\left(x^{\prime}\right)\right] .
\end{aligned}
$$




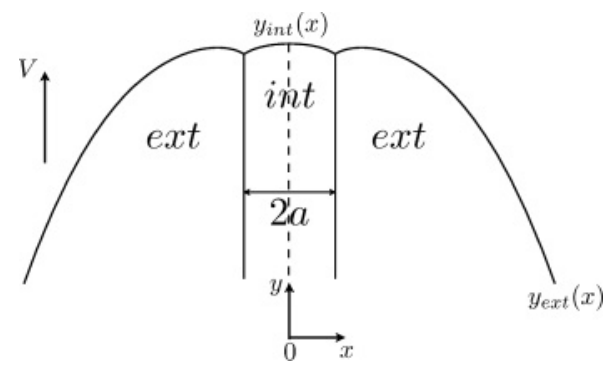

FIG. 1. Geometry of the eutectic two-phase finger growing with steady-state velocity $V$. The solid phases are denoted by ext and int.

Here $\delta_{i, \text { int }}=1$ for $i=$ int and $\delta_{i, \text { int }}=0$ for $i=\operatorname{ext}$;

$$
\Delta_{L}=\left(C_{\text {Lint }}-C_{\text {Lext }}\right) /\left(C_{\text {Sext }}-C_{\text {Lext }}\right)>0
$$

represents the usual driving force in eutectics;

$$
t=-\left(C_{\text {Lint }}-C_{\text {Sint }}\right) /\left(C_{\text {Lext }}-C_{\text {Sext }}\right)>0
$$

is the ratio of the miscibility gaps;

$$
\kappa\left[y_{i}(x)\right]=-\frac{d^{2} y_{i}}{d x^{2}}(x)\left[1+\left(\frac{d y_{i}}{d x}(x)\right)^{2}\right]^{-3 / 2}
$$

is the curvature, which is assumed to be positive for the pattern exhibited in Fig. 1;

$$
\Delta_{\infty}=\left(C_{\text {Lext }}-C_{\infty}\right) /\left(C_{\text {Lext }}-C_{\text {Sext }}\right)>0
$$

represents how "deep" the system is in the two-phase region of the exterior phase. We consider for simplicity that the capillary length $d_{0}$ is the same for both solid-liquid interfaces and that at the triple junction $x=1$, Young law reads $d y_{\text {int }} / d x=-1$, and $d y_{\mathrm{ext}} / d x=1$. Provided that $y_{i}(x)=y_{i}(-x)$, the symmetrized Green's function $g$ is defined as follows:

$$
g\left(x, y ; x^{\prime}, y^{\prime}\right)=\exp \left[P \lambda\left(y-y^{\prime}\right)\right]\left[K_{0}\left(P \lambda \eta^{+}\right)+K_{0}\left(P \lambda \eta^{-}\right)\right],
$$

with $K_{0}$ the modified Bessel function of zeroth order and where $\eta^{ \pm}=\sqrt{\left(x \mp x^{\prime}\right)^{2}+\left[y-y^{\prime}\right]^{2}}$ and the derivative $g^{\prime}$ as

$$
\begin{aligned}
& g^{\prime}\left(x, y ; x^{\prime}, y^{\prime}\right) \\
& \quad=\exp \left[P \lambda\left(y-y^{\prime}\right)\right]\left[K_{0}\left(P \lambda \eta^{+}\right)+f^{+}\left(x, y ; x^{\prime}, y^{\prime}\right) K_{1}\left(P \lambda \eta^{+}\right)\right. \\
& \left.\quad+K_{0}\left(P \lambda \eta^{-}\right)+f^{-}\left(x, y ; x^{\prime}, y^{\prime}\right) K_{1}\left(P \lambda \eta^{-}\right)\right],
\end{aligned}
$$

with $K_{1}$ the modified Bessel function of first order, and $f^{ \pm}\left(x, y ; x^{\prime}, y^{\prime}\right)=\left[-\left(x \mp x^{\prime}\right) d y / d x+y-y^{\prime}\right] / \eta^{ \pm}$.

From Eq. (1), one identifies two important control parameters. First, $\Delta_{\infty}$ is mainly determined by $C_{\infty}$ and is related

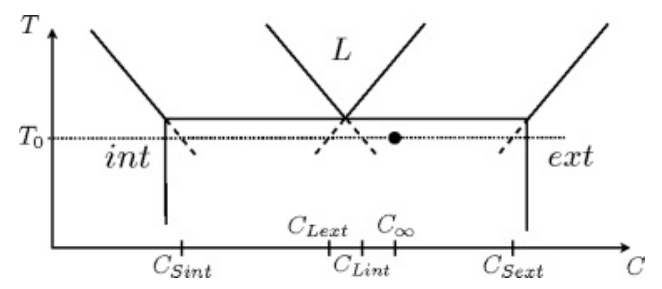

FIG. 2. Eutectic phase diagram. The solid phases are denoted by ext and int and the liquid phase by $L$. The operating point, indicated by the black circle, lies in the liquid-ext two-phase region. to the Peclet number $P$ through the Ivantsov relation, which reads here

$$
\begin{aligned}
\Delta_{\infty} & =\frac{P \lambda}{\pi} \int_{0}^{\infty} d x g\left(x,-\lambda x^{2} / 2 ; x^{\prime},-\lambda x^{\prime 2} / 2\right) \\
& =\sqrt{\pi P} \exp (P) \operatorname{erfc}(\sqrt{P}) .
\end{aligned}
$$

Second, $\Delta_{L}$ is uniquely defined by the temperature of the phase transformation and is proportional to the difference of concentration in the liquid at the two solid-liquid interfaces. One should notice that for the lamellar solidification of eutectic alloys, the lamellae spacing, which fixes the length scale on which the concentration field evolves, is inversely proportional to the square root of the velocity [16]. Since by conservation of mass at the moving solid-liquid interfaces the velocity is proportional to $\Delta_{L}$ divided by such a distance, one finds that the velocity of this lamellar growth is proportional to $\Delta_{L}^{2}$. Then, an invariant quantity is the square of the lamellae spacing times the velocity.

Results. We now present our results, that is, the solution of the equations exhibited above. Solving the equations presented above consists of finding the locus of the interfaces and finding the additional two unknowns, which are $\rho$ and $a$. The velocity $V$ is given by the relation $P=V \rho / 2 D$. The two unknowns $\rho$ and $a$ are determined by the addition of two constraints (one for each interface) at the triple junction given by Young's law. In this respect, let us make two brief comments. First, for the steady-state growth of "multiplets" (patterns with several interior lamellae which are also observed in [14]), the two additional constraints provided by Young's law at each additional triple junction determine the two coordinates of the triple junction. Second, a eutectoid dendrite was theoretically predicted in [5], each side of this dendrite consisting of a different phase, and only one length scale $\rho$ was present in the system. We believe that this solution was a result of the assumed high symmetry (eutectoid composition with a completely symmetric phase diagram), and that this solution does not exist for a more generic system.

We take $t=1$, and we vary $\Delta_{L}$ and $P$. In Fig. 3, the variation of the dimensionless velocity $V d_{0} / 2 D$ versus $\Delta_{L}$ is shown on a logarithmic plot. One clearly observes that, for several orders of magnitude, $V d_{0} / 2 D$ is proportional to

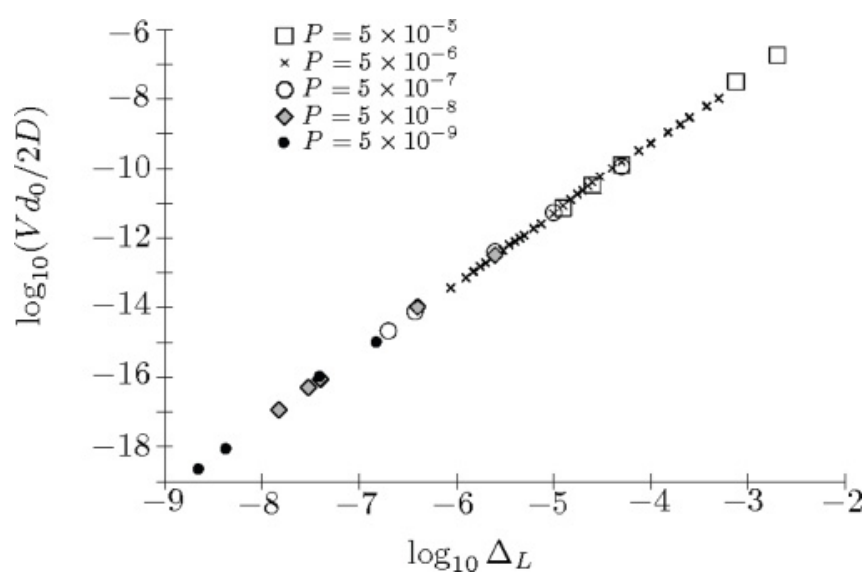

FIG. 3. Dimensionless velocity $V d_{0} / 2 D$ as a function of $\Delta_{L}$ for different values of $P$. 


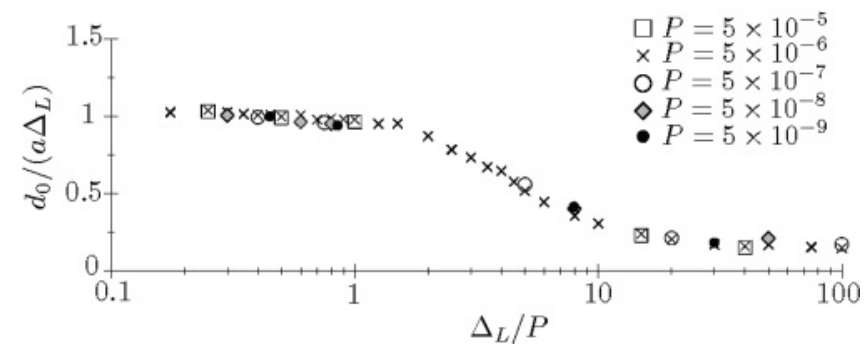

FIG. 4. Plot of $d_{0} /\left(a \Delta_{L}\right)$ versus $\Delta_{L} / P$.

$\Delta_{L}^{2}$ (more precisely, $V d_{0} / 2 D \simeq 0.05 \Delta_{L}^{2}$ ) whatever the value of $P$. Since $V \rho / 2 D=P, \rho / d_{0}$ is proportional to $P / \Delta_{L}^{2}$ (more precisely, $\rho / d_{0} \simeq 20 P / \Delta_{L}^{2}$ ). Concerning the second length scale of the problem $a$, we present in Fig. 4 the variation of $d_{0} /\left(a \Delta_{L}\right)$ versus $\Delta_{L} / P$ for different values of $P$ (from $5 \times 10^{-5}$ to $\left.5 \times 10^{-9}\right)$. We observe that $d_{0} /\left(a \Delta_{L}\right)$ only depends on $\Delta_{L} / P$. Furthermore, we can identify, for $\Delta_{L} / P \leqslant$ 1.5, a plateau where $d_{0} /\left(a \Delta_{L}\right) \simeq 1$. For larger ratios $\Delta_{L} / P$, $d_{0} /\left(a \Delta_{L}\right)$ decreases and seems to saturate at $d_{0} /\left(a \Delta_{L}\right) \simeq 0.15$ for $\Delta_{L} / P \geqslant 30$. According to the results presented in Figs. 3 and 4 , the ratio of the two length scales of the two-phase finger is $\lambda \simeq 0.05 \Delta_{L} /\left(P d_{0} / a \Delta_{L}\right)$ and it only depends on $\Delta_{L} / P$ also. When $d_{0} / a$ is proportional to $\Delta_{L}$ (for $\Delta_{L} / P \leqslant 1.5$ and $\left.\Delta_{L} / P \geqslant 30\right), \lambda$ is proportional to $\Delta_{L} / P$. In Figs. 5(a), 5(b), and 5(c), we exhibit the tip region of the two-phase finger for $\lambda=28.9$ when $\Delta_{L} / P=100, \lambda=1.4$ when $\Delta_{L} / P=8$, and $\lambda=0.022$ when $\Delta_{L} / P=0.45$, respectively. The patterns are qualitatively quite different depending on $\lambda$, the ratio of $a$ and $\rho$, which only depends on $\Delta_{L} / P$. Finally, we present in Fig. 5(d) the result of a phase-field simulation of the eutectic two-phase finger growth using the model developed in [17]. The pattern is qualitatively close to the one presented in Fig. 5(b). Here $t=3$ and the driving forces $\Delta_{\infty}=0.667$ (which corresponds to $P \simeq 0.55$ ) and $\Delta_{L}=1.33$ are very large. Hence, we do not expect a precise quantitative agreement with the boundary-integral results presented above. However, the ratio of the length scales $\lambda \simeq 0.35$ is in good qualitative agreement with the boundary-integral results $(\lambda \simeq 0.17)$ for a ratio $\Delta_{L} / P=2.4$. The dimensionless steady-state velocity $V d_{0} / 2 D \simeq 0.026$ is also in a good qualitative agreement if we use $\Delta_{L}=1.33$ in the formula $V d_{0} / 2 D=0.05 \Delta_{L}^{2}$ $(=0.089)$. This phase-field simulation has the advantage to show that the two-phase finger growth that we studied using the boundary-integral technique is a stable steady-state process. (a)

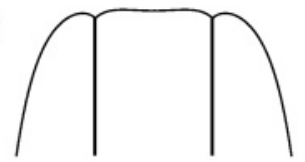

(c)

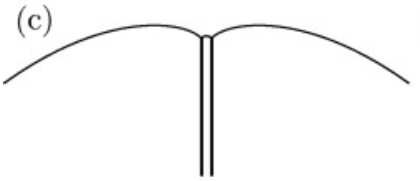

(b)

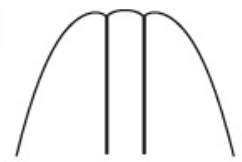

(d)

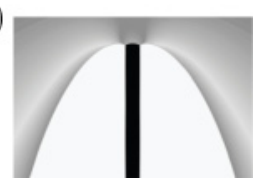

FIG. 5. Tip of the two-phase finger for $\lambda=28.9$ (a), $\lambda=1.4$ (b), and $\lambda=0.022$ (c) and phase-field simulation of the two-phase finger $(d)$.
However, we do not know whether all solutions found from the boundary-integral equations are stable.

Discussion. Let us first discuss the scaling $d_{0} / a \sim \Delta_{L}$ and $\lambda \sim \Delta_{L} / P$ that is suggested by the results of the previous section for $\Delta_{L} / P \leqslant 1.5$ and $\Delta_{L} / P \geqslant 30$. For this purpose we assume the limit $\Delta_{L} \ll 1$ and $P \ll 1$. Taking the small argument limit of the Bessel functions $K_{0}(z) \sim-\ln (z)$ and $K_{1}(z) \sim 1 / z$, Eq. (1) can be written as

$$
\begin{aligned}
& -\frac{1}{2} \Delta_{L} \delta_{i, \text { int }}-\frac{1}{2}\left[1-(t+1) \delta_{i, \text { int }}\right] \frac{d_{0}}{a} \kappa\left[y_{i}^{\prime}\left(x^{\prime}\right)\right] \\
& =+\frac{P \lambda}{\pi} \int_{0}^{1} d x\left[\ln P^{2} \lambda^{2} \eta_{i v}^{+} \eta_{i v}^{-}+t \ln P^{2} \lambda^{2} \eta^{+} \eta^{-}\right] \\
& \quad+\frac{P \lambda}{\pi} \int_{1}^{\infty} d x \ln \frac{\eta_{i v}^{+} \eta_{i v}^{-}}{\eta^{+} \eta^{-}} \\
& +\frac{1}{2 \pi} \int_{0}^{1} d x\left\{\Delta_{L}-t \frac{d_{0}}{a} \kappa\left[y_{\text {int }}(x)\right]\right\}\left(\frac{f^{+}}{\eta^{+}}+\frac{f^{-}}{\eta^{-}}\right) \\
& +\frac{1}{2 \pi} \int_{1}^{\infty} d x \frac{d_{0}}{a} \kappa\left[y_{\mathrm{ext}}(x)\right]\left(\frac{f^{+}}{\eta^{+}}+\frac{f^{-}}{\eta^{-}}\right)
\end{aligned}
$$

with $\eta_{i v}^{ \pm}=\sqrt{\left(x \mp x^{\prime}\right)^{2}+\left(-\lambda x^{2} / 2+\lambda x^{\prime 2} / 2\right)^{2}}$, and using Eq. (2). For $d_{0} / a \sim \Delta_{L}$ and $\lambda \sim \Delta_{L} / P$ all the terms of Eq. (3) are of order $\Delta_{L}$ up to logarithmic corrections. Moreover, the fact that $d_{0} /\left(a \Delta_{L}\right)$ is close to 1 for small ratios $\Delta_{L} / P$ in Fig. 4 can be explained as follows. In the limit $\Delta_{L} / P \rightarrow 0$, we have $\lambda \rightarrow 0$. For the interior interface, the curvature $\kappa\left[y_{\text {int }}(x)\right] \rightarrow 1$, and the left-hand side of Eq. (1) or (3) vanishes with $d_{0} / a \rightarrow$ $\Delta_{L}$ (here $t=1$ ). At the triple junction, the curvature of the exterior interface $\kappa\left[y_{\text {ext }}(x=1)\right] \rightarrow 0$, and the continuity of the concentration field is provided by the Gibbs-Thomson correction of the liquid concentration at the interior interface only. We can thus summarize the latter arguments by scaling laws up to logarithmic corrections:

$$
\frac{a}{d_{0}} \sim \frac{1}{\Delta_{L}}, \quad \frac{\rho}{d_{0}} \sim \frac{P}{\Delta_{L}^{2}} \sim\left(\frac{\Delta_{\infty}}{\Delta_{L}}\right)^{2}, \quad \frac{V d_{0}}{2 D} \sim \Delta_{L}^{2} .
$$

These scaling relations hold for $\Delta_{L} / P \ll 1$ and for $\Delta_{L} / P \gg$ 1 according to our calculations. However, we do not know which mechanism is responsible for the cross-over between these two regimes (see Fig. 4). The parameter space for the description of the two-phase finger growth is a plane (described by $\Delta_{L}$ and $P$ ) and the situation is more complex than in the case of a single control parameter. Indeed, different physical processes may be involved in the selection of the patterns shown in Fig. 5, where $a / \rho$ varies by three orders of magnitude. However, Figs. 3 and 4 demonstrate that fixing the ratio $\Delta_{L} / P$ leaves a one parameter problem with a fixed value of $a / \rho$. We note that, while the scaling relations presented in Eq. (4) are very different from classical dendritic growth, the combination $a^{2} V / d_{0} D$ is still independent of the control parameters. One should notice that this type of invariant quantity is also typical for eutectic lamellar growth.

Figure 3 clearly indicates that the velocity scales as $\Delta_{L}^{2}$, which is the same scaling as for lamellar growth. Surprisingly, the steady-state velocity is thus independent of the global concentration of the alloy $C_{\infty}$. This can be understood by the fact that this scaling law is obtained in the limit of small Peclet number and through a Laplace approximation (replacing the 
Green function and its derivative by their small argument approximation). Indeed, as in the descriptions of lamellar growth, the concentration gradients which are on the scale of the diffusion length are neglected. Here, the information of the global concentration only enters the selection of the asymptotic radius of curvature of the Ivantsov parabola $\rho / d_{0} \sim P / \Delta_{L}^{2}$.

In the limit $P \ll 1$ and $\Delta_{L} \ll 1$, the scaling relations presented in Eq. (4) ensure that $a / d_{0} \sim 1 / \Delta_{L} \gg 1$. Since $\rho / d_{0} \sim$ $P / \Delta_{L}^{2} \sim\left(\Delta_{\infty} / \Delta_{L}\right)^{2}$, the condition $\rho / d_{0} \gg 1$ is fulfilled when $\Delta_{\infty} / \Delta_{L}=\left(C_{\text {Lext }}-C_{\infty}\right) /\left(C_{\text {Lext }}-C_{\text {Lint }}\right) \gg 1$. Hence, when $C_{\infty}$ approaches $C_{\text {Lint }}$, that is, approaches the intersection of the two solid-liquid two-phase regions, $\rho / d_{0}$ becomes of order unity and the existence of such a pattern is questionable. Moreover, the classical lamellar growth is usually observed in this region of the phase diagram. In the other limit $\Delta_{L} / P \ll 1$, the two-phase finger growth may compete with a single-phase dendritic growth (for which the velocity scales as $\alpha P^{2}$, where $\alpha \ll 1$ is linked to the anisotropy coefficient of the surface tension), as suggested in [14].

Note that we performed preliminary phase-field simulations supporting the idea that the two-phase finger growth occurs also in systems such as monotectics or eutectoids (in these systems the diffusion takes place in more than one phase).

Conclusion. In this article, we have presented a scenario for two-phase finger growth in isothermal solidification of eutectic systems. The finger consists of an interior part of one solid phase, and an exterior part of another solid phase. The interior solid phase takes the shape of a lamella parallel to the direction of the velocity, and the exterior part has parabolic Ivantsov asymptotics. We study this dendritic solution in the framework of a boundary-integral method and make a complementary phase-field validation of the stability of the pattern. The deviations from the eutectic temperature and from the eutectic concentration provide two independent control parameters: $\Delta_{\infty}$, which is related to the Peclet number $P$ through the Ivantsov relation, and $\Delta_{L}$. From our results, the steady-state velocity scales as $\Delta_{L}^{2}$. This is the scaling law for the velocity of the classical lamellar growth. The radius of curvature of the asymptotic Ivantsov parabola $\rho$ varies as $P / \Delta_{L}^{2}$. On the other hand, our results show that the ratio of $\rho$ and the width of the interior lamella of the two-phase finger $a$ depends on $\Delta_{L} / P$. Moreover, we propose a scaling relation for $a$ $\left(\sim 1 / \Delta_{L}\right)$ which holds in the two limits $\Delta_{L} \ll P$ and $\Delta_{L} \gg P$. The existence of the two-phase finger was suggested through experimental observations in [14]. Unfortunately, no possible test of our scaling predictions is available through comparison with quantitative experiments. Therefore, we hope that our work will stimulate experimental studies of the two-phase finger described here.

Acknowledgements. We acknowledge the support of the Deutsche Forschungsgemeinschaft under Project No. SPP1296. We are also grateful to M. Plapp for providing the phase-field code and D.E. Temkin for useful discussions.
[1] G. P. Ivantsov, Dokl. Akad. Nauk SSSR 68, 567 (1947).

[2] D. Kessler, J. Koplik, and H. Levine, Adv. Phys. 37, 255 (1988).

[3] E. A. Brener and V. I. Mel'nikov, Adv. Phys. 40, 53 (1991).

[4] D. E. Temkin, Abstract of ICASP, June 7-10 2005, Stockolm, Sweden (KTH, Stockolm, 2005).

[5] E. A. Brener, C. Hüter, D. Pilipenko, and D. E Temkin, Phys. Rev. Lett. 99, 105701 (2007).

[6] D. Pilipenko, E. A. Brener, and C. Hüter, Phys. Rev. E. 78, 060603 (2008).

[7] J. D. Hunt and K. A. Jackson, Trans. Soc. Min. Eng. AIME 236, 843 (1966).

[8] M. Ginibre, S. Akamatsu, and G. Faivre, Phys. Rev. E 56, 780 (1997).
[9] A. Karma and A. Sarkissian, Met. Trans. A 27, 635 (1996).

[10] M. Plapp, J. Cryst. Growth 303, 49 (2007).

[11] G. Faivre and J. Mergy, Phys. Rev. A 45, 7320 (1992).

[12] K. Kassner and C. Misbah, Phys. Rev. A 44, 6533 (1991).

[13] S. Akamatsu, M. Perrut, S. Bottin-Rousseau, and G. Faivre, Phys. Rev. Lett. 104, 056101 (2010).

[14] S. Akamatsu and G. Faivre, Phys. Rev. E 61, 3757 (2000).

[15] K. Kassner and C. Misbah, Phys. Rev. A 44, 6513 (1991).

[16] K. Kassner and C. Misbah, Phys. Rev. Lett. 66, 445 (1991).

[17] R. Folch and M. Plapp, Phys. Rev. E 44, 011602 (2005). 